\title{
Controlling Electrochemical Chaos in the Copper-Phosphoric Acid System
}

\author{
I. Z. Kiss and V. Gáspár* \\ Institute of Physical Chemistry, Kossuth Lajos University, H-4010 Debrecen, P.O. Box 7, Hungary
}

L. Nyikos

Physical Chemistry Laboratory, Central Research Institute for Physics, H-1525 Budapest, P.O. Box 49, Hungary

\section{P. Parmananda}

Facultad de Ciencias, U.A.E.M., Av. Universidad 1001, Col. Chamilpa, C.P. 62210, Cuernavaca, Morelos, Mexico

Received: July 10, $1997^{\otimes}$

\begin{abstract}
Model calculations indicate that chaotic current oscillations during anodic electrodissolution of copper into phosphoric acid may be controlled by applying a simple map-based algorithm. In the experiments, the unstable period-one and period-two orbits embedded in the chaotic attractor have been stabilized by small perturbations of the anodic potential. We present the results of an experimental test for a power law relating the average chaotic transient time to the size of maximum perturbation allowed during control. The reported experimental results are in good agreement with the theoretical predictions by Ott, Grebogi, and Yorke.
\end{abstract}

\section{Introduction}

Due to advances in the field of nonlinear dynamics, the interest in electrochemical systems exhibiting a variety of nonlinear phenomena ranging from simple oscillations to chaos has been renewed. Comprehensive reviews on electrochemical reaction dynamics have been published recently by Hudson and Tsotsis $^{1}$ and Koper. ${ }^{2}$ Despite the prevalence of chaos in electrochemical systems, most of the experiments are designed to avoid parameter ranges where such "complications" may arise. However, since the advent of the celebrated OttGrebogi-Yorke (OGY) theory ${ }^{3}$ of chaos control, it has been realized that operating in a chaotic regime may be even advantageous.

Following OGY several control methods have been developed and applied to control chaos in different experimental settings, e.g., magneto-elastic and hydrodynamic systems, electric circuits, lasers, chemical reactions, and tissues of heart and brain in vitro. For a review on different control strategies and their applications we refer to a recent paper by Shinbrot. ${ }^{4}$ The most often applied OGY algorithm is based on the sensitivity of a chaotic system to initial conditions and utilizes the short-term predictability of the deterministic dynamics: a desired periodic orbit embedded in the chaotic attractor is being stabilized by applying small, time-dependent perturbations to a control parameter.

Surprisingly, there are only few experimental examples of chaos control in chemical systems. ${ }^{5-9}$ This might be due to the hardship involved in such experiments because of the high level of internal noise and the relatively large period of chaotic oscillations in the homogeneous chemical systems studied so far. $^{5-7}$ Electrochemical systems, on the other hand, seem to be an ideal playground for the chaotically inclined. The reproducibility of electrochemical experiments is good, the level of internal noise is low, the period of chaotic oscillations may be very short, the behavior can be monitored by simple current or voltage measurements, and the control parameter (potential

\footnotetext{
* To whom correspondence should be addressed.

${ }^{\otimes}$ Abstract published in Advance ACS Abstracts, October 15, 1997.
}

or current) is easily attainable. The idea has been put into practice first by Parmananda et al. ${ }^{8,9}$ They reported on controlling the chaotic current oscillations during the anodic dissolution of a rotating copper-disk electrode in sodium acetateglacial acetic acid buffer.

In this paper, we report the control of chaotic current oscillations in the copper-phosphoric acid system under potentiostatic conditions. The dynamics of this system has been extensively studied earlier by Schell et al..$^{10-12}$ They showed that for an appropriate range of control parameters the system exhibits a variety of interesting behavior, such as simple oscillations, period-doubling cascades, mixed-mode transitions, and chaos. First we attempt to control chaos in a simple skeleton model for the system. The three-variable model, which simulates the dynamics in good agreement with the experiments, has been developed by Koper and Gaspard. ${ }^{13}$ Our aim with the model calculations is to test if a simple proportional feedback (SPF) algorithm introduced by Peng, Petrov, and Showalter ${ }^{14,15}$ can be used to control chaos in this electrochemical system. (In an earlier publication ${ }^{16}$ one of the authors of this paper (P.P.) has studied a somewhat similar model ${ }^{17}$ simulating the chaotic reduction of indium(III) at a hanging mercury electrode in the presence of thiocyanate and found that control in that skeleton model can be achieved by using the SPF algorithm.) After briefly describing the Koper-Gaspard mode $1^{13}$ and the applied map-based strategy, we present the numerical results. Next we attempt to apply the algorithm to the experimental system. We show that the unstable period-one and period-two orbits embedded in the chaotic attractor can, indeed, be stabilized by small perturbations of the anodic potential as control parameter. We then present the results of an experimental test for a power law relating the average chaotic transient time to the size of maximum perturbation allowed during control. We conclude by summarizing the results and discuss the advantages of the studied system for testing different control strategies.

\section{Numerical Studies}

Model. The first-order differential equations of the KoperGaspard model ${ }^{13}$ are given in a dimensionless form, 


$$
\begin{gathered}
\frac{\mathrm{d} e}{\mathrm{~d} t}=\frac{v-e}{r}-m k(e) u \\
\frac{\mathrm{d} u}{\mathrm{~d} t}=-1.25 d^{1 / 2} k(e) u+2 d(w-u) \\
\frac{\mathrm{d} w}{\mathrm{~d} t}=1.6 d(2-3 w+u)
\end{gathered}
$$

where $v$ is the applied (circuit) potential, $e$ is the "true" electrode potential appearing across the interfacial double layer, $r$ is an adjustable series resistance, $d$ is the rotation rate, and $k(e)$ is the heterogeneous rate constant determining the rate of electron transfer. To account for the mass-transport from the solution to the rotating disk a simple two-diffusion-layer model ${ }^{13}$ is applied. Variables $u$ and $w$ are the normalized concentrations of the electroactive species, respectively, in the so-called "surface" and "diffusion" layers, while $m$ is the concentration in the bulk. Equations 1 are studied under potentiostatic conditions with circuit potential $v$ as the control parameter. For monitoring the behavior we use the total current $i=(v-e) / r$ which is an easily measurable function of the system variables. Since the model equations are not stiff, a simple fourth-order Runge-Kutta method with constant time step $(\Delta t=0.01)$ has been applied for integration.

The potential-dependent rate constant $k(e)$ is given by a prototype function,

$$
k(e)=k_{1} \theta^{2}+k_{2} \exp \left[n \alpha\left(e-e^{\mathrm{o}}\right)\right]
$$

where $e^{\mathrm{o}}$ is the dimensionless standard potential, $\alpha$ is the transfer coefficient, and $\theta$ is related to the surface coverage by some electroactive species. The value of $\theta$ is approximated by a sigmoidal function,

$$
\theta=\left\{\begin{array}{ll}
1 & \text { for } e \leq e_{d} \\
\exp \left[-b\left(e-e_{d}\right)^{2}\right] & \text { for } e>e_{d}
\end{array}\right\}
$$

Equations 2 and 3 give rise to a potentially unstable electrochemical flux and also account for the experimentally observed negative slope of the polarization curve in some potential intervals (compare with Figure 3 discussed later).

The dynamics of model eqs $1-3$ have been studied in detail by Koper and Gaspard. ${ }^{13}$ For an appropriate range of parameters, the model exhibits a variety of nonlinear behaviors such as simple oscillations, period-doubling cascades, mixed-mode transitions, and chaos as different control parameters (e.g., circuit potential $v$, rotation rate $d$, and series resistance $r$ ) are systematically varied. The agreement with the experiments by Schell et $a l .^{10-12}$ is remarkable. For the parameter values given in the legend of Figure 1, the system exhibits a simple period-doubling route to chaos. The bifurcation diagram shows the maxima of total current as a function of control parameter $v$. Using the control strategy discussed in the next subsection the chaotic behavior observed at $v=36.74$ has been converted to different periodic oscillations.

Map-Based Algorithm for Controlling Chaos. From the chaotic time series calculated with the just defined parameter values, a return map $x_{n+1}$ vs $x_{n}$ was constructed by plotting the successive maxima of the total current. Since the dynamical behavior is represented by a one-dimensional (1D) next-return map, a single proportional feedback algorithm ${ }^{11,12}$ is applied to controlling chaos. Control is achieved by perturbing the control parameter $v$ such that the fixed point $x_{\mathrm{f}}$ of the map is targeted on each return. (Note that the fixed point corresponds to an unstable periodic orbit since $x_{n+1}=x_{n}$.) In the linear range around the fixed point, the next iterates are given by,

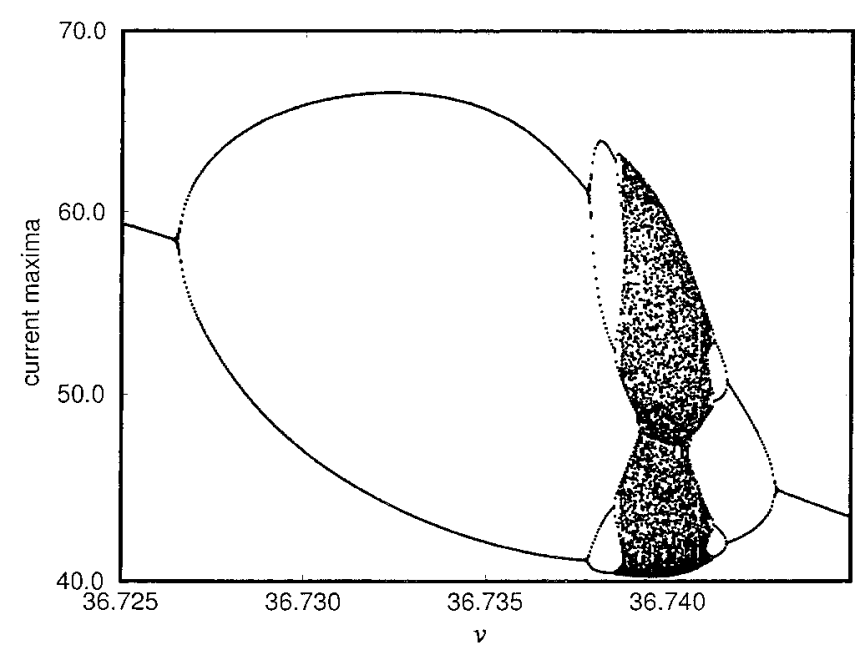

Figure 1. Bifurcation diagram of the skeleton model eqs 1-3. Maxima of anodic current are plotted vs circuit potential $v$ as control parameter. Dimensionless parameters are $r=0.02, m=120, d=0.11915, k_{1}=$ $2.5, k_{2}=0.01, n \alpha=0.5, e^{\mathrm{o}}=30.0, e_{\mathrm{d}}=35.0$, and $b=0.5$.

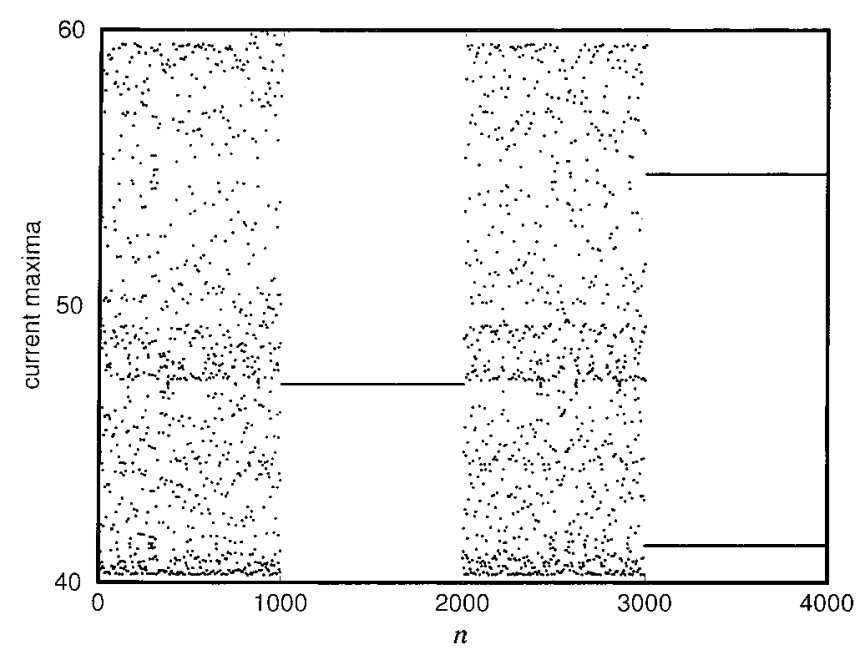

Figure 2. Maxima of anodic current are plotted vs iteration number $n$ over an interval when the control for period-one oscillations is switched on and off followed by the control of period-two unstable orbit. For the uncontrolled system $v=36.74$. Other model parameters are given in Figure 1.

$$
x_{n+1}=x_{\mathrm{f}}(v)+\mu\left[x_{n}-x_{\mathrm{f}}(v)\right]
$$

where $|\mu|>1$ is the slope of the map. When the value of the control parameter is changed from $v$ to $v+\delta v$, the map shifts. For simplicity, we assume that $\mu$ remains constant in a small range $\delta v_{\max }$ around $v$. Applying the control condition to the linearized equation of the shifted map as well, the potential perturbation $\delta v_{n}$ required to place the next iterate on the original fixed point $x_{\mathrm{f}}$ can be calculated by the following control formula,

$$
\delta v_{n}=\frac{\mu}{(\mu-1) g}\left(x_{n}-x_{\mathrm{f}}\right)=K\left(x_{n}-x_{\mathrm{f}}\right)
$$

where $g=\delta x_{\mathrm{f}} / \delta v \neq 0$ is a constant scalar relating the change in the value of the fixed point to the size of the applied perturbation. When control is desired the potential is changed according to eq 5 whenever the maximum current falls within a small range of fixed point $x_{\mathrm{f}}$. If, however, eq 5 requires a large change in the potential such that $\delta v_{n} \geq \delta v_{\max }$, the perturbation is not applied and the system is allowed to relax to its original chaotic attractor until the current maxima again visits the neighborhood of the fixed point.

The parameter values in control formula 5 have been determined from chaotic time series calculated at $v_{1}=36.7400$ 


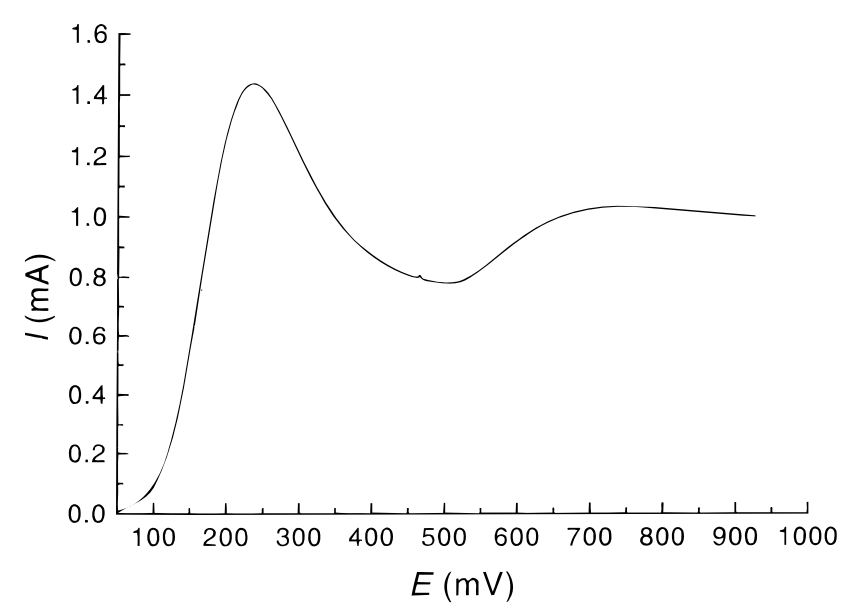

Figure 3. Polarization curve (current vs true electrode potential) of a copper RDE in phosphoric acid electrolyte. The solution resistance $R_{\mathrm{s}}$ is $72 \Omega$, rotation rate is $1800 \mathrm{rpm}$, and scan rate is $10 \mathrm{mV} \mathrm{s}^{-1}$. The small wiggling of the curve near $450 \mathrm{mV}$ is due to an unidentified instability of the circuit.

and $v_{2}=36.7399$ constant potentials applied to the uncontrolled system. The fixed points $x_{\mathrm{f} 1}=47.13$ and $x_{\mathrm{f} 2}=47.21$, and the slopes of the maps $\mu_{1}=-1.98$ and $\mu_{2}=-2.02$, were calculated by linear least-squares fit to the next return data collected in a small range around the fixed points. Note that the small change in the potential resulted in only negligible change in the slope. This procedure allowed us to calculate the value of $g=-800$ and $K=-8.31 \times 10^{-4}$.

Controlling Chaos in the Numerical Model. Figure 2 shows the current maxima over an interval when the control algorithm to stabilize period-one oscillations has been switched on and off. As targeting is never perfect due to errors in determinations of fixed point $x_{\mathrm{f}}$ and constant $K$, continuous corrections to the control parameter $v$ are required to maintain control.

The same control strategy can be implemented to stabilize any desired unstable periodic orbit embedded in the chaotic attractor. For example, in Figure 2 we also show the control of period-two oscillations. The parameters in eq 5 have been recalculated by the earlier algorithm but using the second return maps $x_{n+2}$ vs $x_{n}$ of the uncontrolled system at $v_{1}=36.7400$ and $v_{2}=36.7399$. The fixed points $x_{\mathrm{f} 1}=54.78$ and $x_{\mathrm{f} 2}=55.04$ and the slope of the map $\mu_{1}=-2.25$ give us the constants $g=$ -2600 and $K=-2.66 \times 10^{-4}$. To achieve control of periodtwo, however, the size of perturbation has to be recalculated only at every second return to the map. The successful application of a simple proportional feedback algorithm to controlling chaos in the skeleton model has greatly motivated us to try out the same strategy in the experimental system as well.

\section{Experimental Section}

Experimental Setup. Experiments were performed by using a standard three-electrode electrochemical cell equipped with a rotating disk electrode (RDE), a Radelkis $\mathrm{OH}-0933 \mathrm{P}$ saturated calomel electrode (SCE) as reference, and a Radelkis OH-9437 Pt-sheet counter electrode (area $5 \mathrm{~cm}^{2}$ ). A small cut of a copper rod (5 mm diameter) was fixed in the Teflon holder of the RDE apparatus (EDI 101, Radiometer). Before each experiment the surface of the copper sample ( $99.99 \%$ purity) has been freshly polished by a series of wet sanding. The cell contained $70 \mathrm{~cm}^{3}$ ortho-phosphoric acid (85\%, Merck or Spektrum-3D) and was thermostated at $-17.5 \pm 0.1^{\circ} \mathrm{C}$ by using a Lauda RM6B. All measurements reported in this paper were made with the depth of the rotating copper disk electrode (penetrating the solution) and the distance between it and the reference electrode set at 10 and $25 \mathrm{~mm}$, respectively. The distance between the copper and platinum electrodes was set at $15 \mathrm{~mm}$.

The cell was connected to a computer-controlled potentiostat (Electroflex EF451). The potential between the RDE and SCE was measured and set with a resolution of 0.2 and $0.01 \mathrm{mV}$, respectively, for simple potentiostatic and chaos control experiments. In this report, all potentials are given with respect to the SCE. The output current signal between the RDE and platinum electrode was fed into a 12-bit A/D converter built in the potentiostat. Sampling frequencies of 100 and $200 \mathrm{~Hz}$ were applied, respectively, for data acquisition and control experiments. The digitized current data were stored on the PC for on-time calculations and later plotting. The rotation rate was controlled via a 12-bit D/A converter (Labcard PCL-818L) also built in the PC.

Procedures. To remove the oxide layer from the surface of the copper electrode, the potential is first set to $500 \mathrm{mV}$ for 2 min and then swept in several cycles between 0 and $750 \mathrm{mV}$ with a scan rate of $10 \mathrm{mV} \mathrm{s}^{-1}$. Next the series (ohmic) resistance $R_{\mathrm{S}}$ of the solution is determined from impedance measurements in the frequency range of $5-10 \mathrm{kHz}$ at $40 \mathrm{mV}$ rest potential. The value of $R_{\mathrm{s}}$ is calculated by linearly extrapolating the impedance spectrum to infinite frequency where the imaginary part of the total impedance is zero. For details on application of impedance spectroscopy to this system we refer to our earlier report. ${ }^{18}$ The series resistance of a given system has been determined by averaging the results of at least three impedance measurements. If the $R_{\mathrm{S}}$ value was out of the range between 70.0 and $74.0 \Omega$, or the voltammogram of the system was much different from that shown in Figure 3, the copper electrode was replaced with a new sample. We note that when the treatment was successful the electrode surface has been electropolished homogeneously, while in case of failure few concentric rings of different "gray" levels could be always observed on the copper electrode.

In agreement with the results of numerical studies by Koper and Gaspard, ${ }^{13}$ we have found that the chaotic range of the experimental bifurcation diagrams - spanned by the rotation rate and circuit potential-is widening and shifts to lower rotation rates as the total ohmic resistance of the circuit is increased. After a trial-and-error procedure we decided to connect an external resistance of $130 \Omega$ in series with the copper electrode resulting in a total resistance $R_{\Omega}$ of 200-204 $\Omega$ for all experiments reported here.

Before a series of experiments with a successfully treated electrode the potential was set to $540 \mathrm{mV}$ for $1-2 \mathrm{~h}$. During this period the current oscillations have generally stabilized. With this procedure the bifurcation diagrams were reproducible within $\pm 100 \mathrm{rpm}$ and $\pm 10 \mathrm{mV}$. All bifurcation diagrams were measured by increasing the anodic potential. After a stepwise potential jump a waiting period of $60 \mathrm{~s}$ was applied during which the current oscillations stabilized. Minima and maxima of current were determined from data collected in the next $60 \mathrm{~s}$ interval.

According to literature accounts, ${ }^{19}$ oxygen does not affect the dynamics of the copper-phosphoric acid system. We have also found that bubbling nitrogen through the cell does not modify the bifurcation diagram. Therefore, no special care was taken to prevent the diffusion of air-oxygen into the cell.

\section{Experimental Results}

Conditions for Period-Doubling Route to Chaos. In the numerical section, the SPF algorithm was successfully applied 
TABLE 1: Dynamical States and Bifurcations in the Copper-Phosphoric Acid System ${ }^{a}$

\begin{tabular}{cl}
\hline $\begin{array}{c}\text { fixed rotation } \\
\text { rate }(\mathrm{rpm})\end{array}$ & \multicolumn{1}{c}{$\begin{array}{c}\text { dynamical states and } \\
\text { bifurcations in the potential range } \\
\text { from } 430 \text { to } 520 \mathrm{mV}\end{array}$} \\
\hline 600 & $\mathrm{SS}$ \\
700 & $\mathrm{SS} \rightarrow \mathrm{P} 1 \rightarrow \mathrm{MMO} \rightarrow \mathrm{SS}$ \\
800 & $\mathrm{SS} \rightarrow \mathrm{P} 1 \rightarrow \mathrm{MMO} \rightarrow \mathrm{SS}$ \\
1550 & $\mathrm{SS} \rightarrow \mathrm{P} 1$ \\
1600 & $\mathrm{SS} \rightarrow \mathrm{P} 1 \rightarrow \mathrm{P} 2 \rightarrow \mathrm{P} 1$ \\
1650 & $\mathrm{SS} \rightarrow \mathrm{P} 1 \rightarrow \mathrm{P} 2 \rightarrow \mathrm{P} 1$ \\
1700 & $\mathrm{SS} \rightarrow \mathrm{P} 1 \rightarrow \mathrm{P} 2 \rightarrow \mathrm{P} 1$ \\
1800 & $\mathrm{SS} \rightarrow \mathrm{P} 1 \rightarrow \mathrm{P} 2 \rightarrow \mathrm{P} 4 \rightarrow \mathrm{P} 2 \rightarrow \mathrm{P} 1$ \\
1850 & $\mathrm{SS} \rightarrow \mathrm{P} 1 \rightarrow \mathrm{P} 2 \rightarrow \mathrm{P} 4 \rightarrow \mathrm{P} 2 \rightarrow \mathrm{P} 1$ \\
1900 & $\mathrm{SS} \rightarrow \mathrm{P} 1 \rightarrow \mathrm{P} 2 \rightarrow \mathrm{C} \rightarrow \mathrm{P} 2 \rightarrow \mathrm{P} 1$ \\
1950 & $\mathrm{SS} \rightarrow \mathrm{P} 1 \rightarrow \mathrm{P} 2 \rightarrow \mathrm{C} \rightarrow \mathrm{P} 2 \rightarrow \mathrm{P} 1$ \\
2000 & $\mathrm{SS} \rightarrow \mathrm{P} 1 \rightarrow \mathrm{P} 2 \rightarrow \mathrm{C} \rightarrow \mathrm{P} 3 \rightarrow \mathrm{C} \rightarrow \mathrm{P} 2 \rightarrow \mathrm{P} 1$
\end{tabular}

${ }^{a}$ At a fixed rotation rate, the applied potential is increased from 430 to $520 \mathrm{mV}$ with a stepsize of $1.0 \mathrm{mV}$. The total series resistance is $200 \Omega$. Annotations are as follows: steady state: SS, period-one: P1, period-two: P2, period-three: P3, period-four: P4, mixed-mode oscillations: $\mathrm{MMO}$, and chaos: $\mathrm{C}$.

to control chaos which originated from a period-doubling cascade of bifurcations. To find the right conditions for the same type of chaos in the experimental system, we first explored the parameter space in detail. Two parameters, the rotation rate and the applied potential have been systematically varied.

Table 1 summarizes the dynamical states exhibited by a given copper-phosphoric acid system $\left(R_{\Omega}=200 \Omega\right)$ at several different rotation rates when the applied potential was increased with a stepsize of $1 \mathrm{mV}$ from 430 to $520 \mathrm{mV}$. At $600 \mathrm{rpm}$ only steady-states have been found. At larger rotation rates, however, complex waveforms developed. The current oscillations observed in the range between 1600 and $2000 \mathrm{rpm}$ clearly indicated a period-doubling route to chaos. At $2000 \mathrm{rpm}$ we detected a period-three window indicative of chaos. As the rotation rate was increased even further (not shown in Table 1), more and more chaotic bands appeared in the bifurcation diagram. These bands were separated by regions of mixedmode transitions. The overall behavior is the same as that found by Schell et al. ${ }^{10-12}$ at much larger rotation rates (approximately $4000 \mathrm{rpm}$ ). With the application of an external resistance in our experiments, the bifurcation diagram has been shifted to lower rotation rates hence our system is more "user-friendly" from a technical point of view.

Our goal was to control chaotic current oscillations at such rotation rates where chaos develops through simple periodicdoubling bifurcations. Figure 4 shows both the minima and maxima of current oscillations for such sequence of bifurcations as the potential is varied. The chaotic region in the middle of the diagram spans over an approximately $3 \mathrm{mV}$ range. When control is attempted, the anodic potential of the uncontrolled system is always set near the middle of the chaotic range making sure that the system is not shifted out of chaos when maximum of $0.5 \mathrm{mV}$ potential perturbations are applied during control.

Chaos Control. Preliminary experiments indicated that the algorithm applied in the numerical studies should be slightly modified. In the control experiments instead of current maxima, which are hard to measure with great accuracy, we rather used the next-return values on a Poincaré section of the reconstructed chaotic attractor as defined in the legend of Figure 5. The correlation dimension of the chaotic attractor is $2.25 \pm 0.1$. This value is in good agreement with that determined by Hudson et $a l .{ }^{20}(2.3 \pm 0.2)$ at quite different experimental conditions $(4400$ rpm, no external resistance, and $20^{\circ} \mathrm{C}$ ). Since the attractor is almost two-dimensional, the dynamics of the copper-phosphoric acid system may be represented by a 1-D next return map

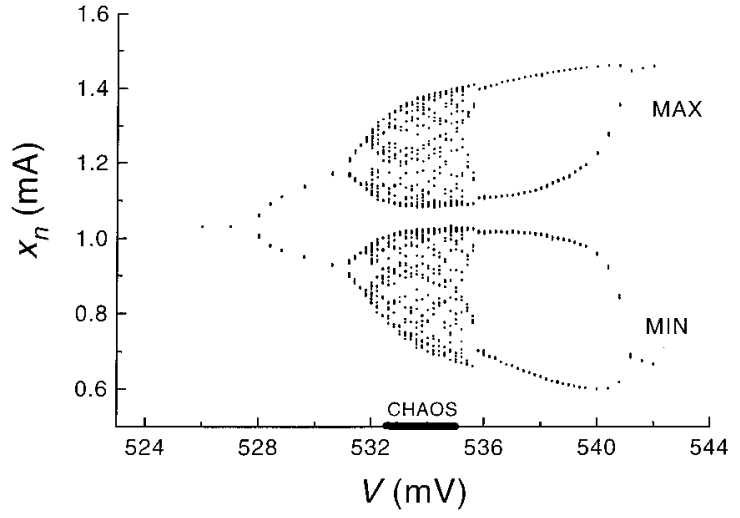

Figure 4. Bifurcation diagram of the copper RDE-phosphoric acid system showing both maxima and minima of current oscillations as a function of the applied anodic potential. Total ohmic resistance of the circuit is $202 \Omega$ and rotation rate is $1850 \mathrm{rpm}$.

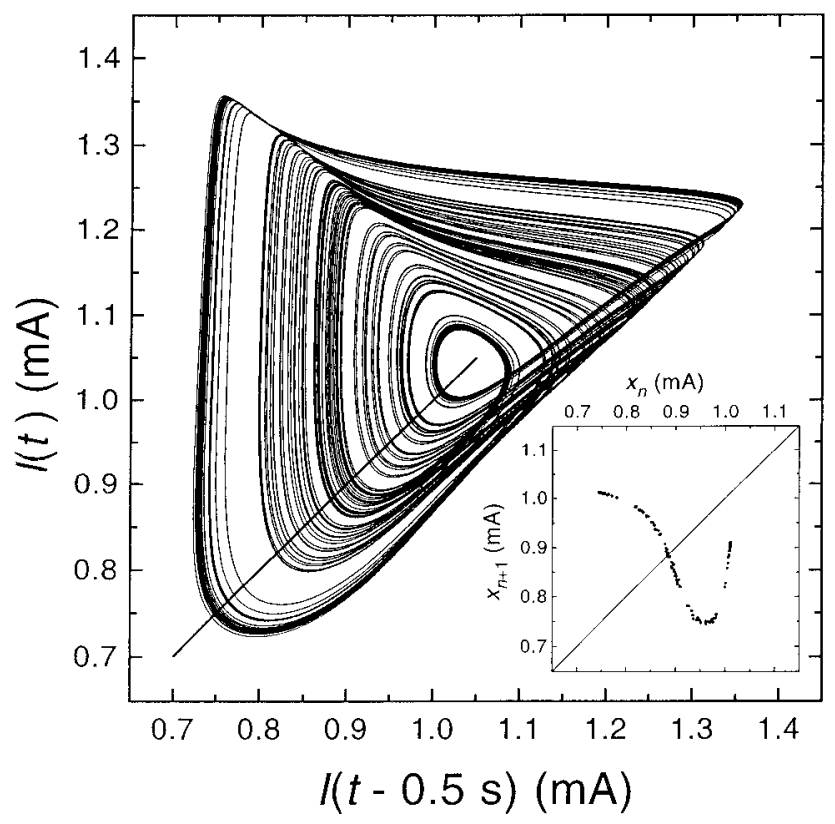

Figure 5. Reconstructed chaotic attractor of the copper-phosphoric acid system in the phase space of time-delay coordinates $I(t)$ and $I(t-$ $0.5 \mathrm{~s}$ ), where $t$ is real time (s). The next return map $x_{n+1}$ vs $x_{n}$ has been generated by using successive current values on the Poincare section (thick line) defined as $I(t)=I(t-0.5 \mathrm{~s})$ and $d I(t) / d t>0$. The applied anodic potential is $532.0 \mathrm{mV}$, total ohmic resistance is $202 \Omega$, and rotation rate is $1800 \mathrm{rpm}$.

(shown in the inset). Thus we hoped that the SPF algorithm can be applied to controlling chaos in the experimental system as well.

The value of control constant $K$ in eq 5 has been determined by using the next return data shown in Figure 6 . Note that the slope did not change significantly by shifting map. The slope of the maps given in the legend were calculated by linear leastsquares fit. This gave us $K=-17 \pm 4 \mathrm{mV} / \mathrm{mA}$ for periodone control. In Figure 7 we plotted time series current data for an interval when control for stabilizing period-one oscillations has been switched on and off. Note that the longer the control is on, the smaller is the potential perturbation $\delta V_{n}$ that should be applied at the successive returns to the Poincaré section. Toward the end of the control interval the perturbation is less than $0.1 \mathrm{mV}$ (approximately $0.02 \%$ of the applied potential). We may say that instead of shifting the system out of the chaotic range, we only forced the trajectories to remain in the neighborhood of the unstable periodic orbit. Consequently, when control is turned off, the behavior is almost periodic for a couple of 


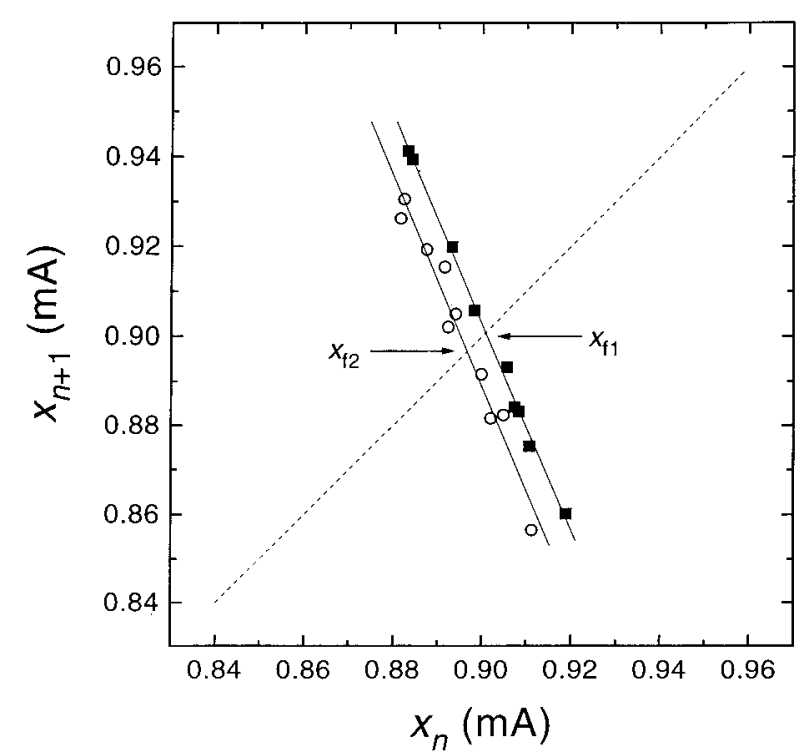

Figure 6. Return maps for parameter values $V_{1}=532.0$ (squares) and $V_{2}=532.1 \mathrm{mV}$ (circles) with fixed points of $x_{\mathrm{f} 1}=0.9008 \pm 0.0002$ and $x_{\mathrm{f} 2}=0.8966 \pm 0.0002 \mathrm{~mA}$, respectively. The slopes $\mu_{1}$ and $\mu_{2}$ are $-2.31 \pm 0.5$ and $-2.34 \pm 0.5$, respectively. Experimental conditions are given in Figure 5.

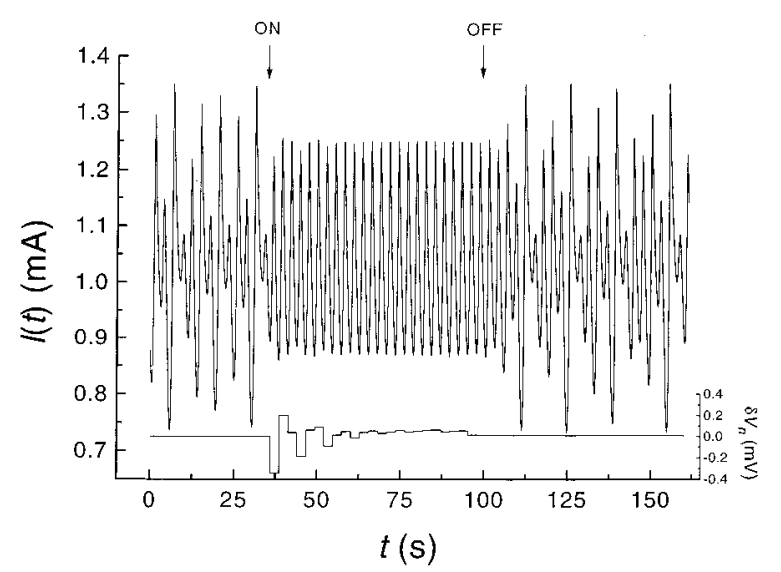

Figure 7. Current (left axis) vs time for an interval when control for stabilizing period-one oscillations has been switched on at $35.2 \mathrm{~s}$ and then switched off at $99.3 \mathrm{~s}$. The control constant $K$ for stabilizing periodone oscillations is $-17 \pm 4 \mathrm{mV} / \mathrm{mA}$, while $\delta V_{\max }$ was set to $0.5 \mathrm{mV}$. The potential perturbations $\delta V_{n}$ (right axis) applied at the successive returns to the Poincaré section are shown in the insert. For experimental conditions see Figure 5.

cycles but then the trajectories wind off to land at the stable chaotic attractor again.

The same control strategy has been implemented to stabilize period-two oscillations shown in Figure 8. The value of control constant $K$ has been determined as before but using secondreturn maps $x_{n+2}$ vs $x_{n}$ of the uncontrolled system measured at two, slightly different potential values $(527.0$ and $527.2 \mathrm{mV})$. The inset shows the next return map of the uncontrolled system at $527 \mathrm{mV}$. Superimposed on the map are the next-return values while control is being implemented with control constant $K=$ $-11 \mathrm{mV} / \mathrm{mA}$. Note that the size of perturbation is recalculated only at every second return to the map. We point out that during period-two control the perturbations are generally larger (the value of $\delta V_{n}$ varied within a range of $\pm 0.25 \mathrm{mV}$ ) than those applied during period-one control. This can be interpreted by the fact that after two cycles (one small and one large) the trajectory is repelled to a greater distance from the unstable orbit.

Experiments for stabilizing period-one or period-two oscillations have been repeated several times. For every new session

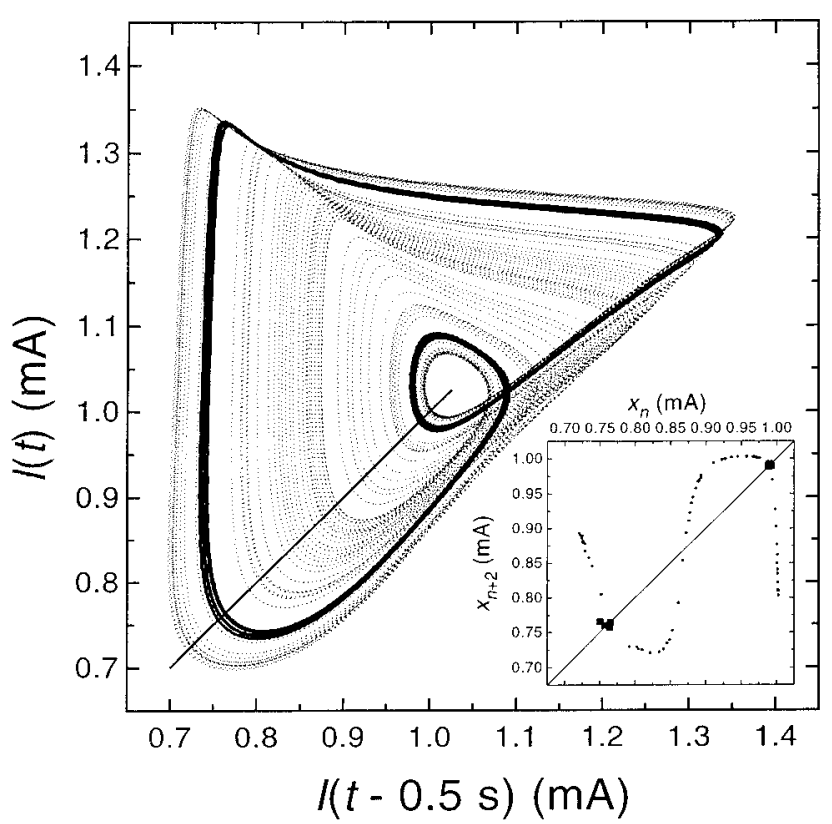

Figure 8. Stabilized period-two orbit $\left(x_{\mathrm{f}}=0.7726 \mathrm{~mA}\right)$ embedded in the chaotic attractor (dots). The next-return map $x_{n+2}$ vs $x_{n}$ has been generated by using successive current values on the Poincaré section (thick line) as defined in Figure 5. The control parameter $K$ for periodtwo control is $-11 \mathrm{mV} / \mathrm{mA}$ and $\delta V_{\max }$ is $0.5 \mathrm{mV}$. Superimposed on the map are the next-return values (filled squares) while control is being implemented. The applied anodic potential in the uncontrolled system is $527 \mathrm{mV}$, total ohmic resistance is $202 \Omega$, and rotation rate is 1900 rpm.

we have determined the actual value of $K$ as described earlier. The $K$ values always varied between -10 and $-30 \mathrm{mV} / \mathrm{mA}$, most probably, due to small changes (noise) in external parameters such as rotation rate, temperature, etc. We should also note that determination of $K$ may also be somewhat inaccurate because of a continuous, though small shift of the next-return map. This shift is unavoidable and originates from the drift of the closed system toward its equilibrium. Fortunately, the SPF algorithm is so robust that a small error in the value of $K$ does not really matter. In addition, as perturbations are applied at every (or every second) return to the map, the imperfect targeting is repeatedly corrected (to a certain limit) by the algorithm itself.

Occasionally, we have encountered failures during the control process. This generally happened when after many hours of experimentation the dynamics of a given system changed into what can be described as "banded chaos" thus the fixed point of the next-return map was not visited for extremely long intervals.

Chaotic Transient Time vs Maximum Perturbation. Knowing something about the nature of chaotic transients after the control algorithm has been turned on is of great interest, especially, from the point of view of real-world applications. For example, it would be desirable to be able to predict the length of transient period $\tau$, the time to achieve control. Noticing that during these transients the orbit is similar to those of the uncontrolled chaotic attractor, and taking also into account that the time to achieve control depends sensitively on the initial conditions, OGY formulated a simple power law, ${ }^{3}$

$$
\langle\tau\rangle \propto\left(\delta V_{\max }\right)^{-\gamma}
$$

relating the average length of chaotic transients $\langle\tau\rangle$ to the size of maximum perturbation $\delta V_{\max }$. The value of the exponent $\gamma$ is given in terms of eigenvalues $\lambda_{\mathrm{s}}$ and $\lambda_{\mathrm{u}}$, respectively, in the 
stable and unstable directions of the fixed point $x_{\mathrm{f}}$ by the following formula,

$$
\gamma=1+\frac{1}{2} \frac{\ln \left|\lambda_{u}\right|}{\ln \left(1 /\left|\lambda_{\mathrm{s}}\right|\right)}
$$

Numerical experiments for testing eq 6 have been carried out by using the Henon map. ${ }^{3}$ After calculating the average length of chaotic transients determined at large number of randomly selected initial conditions for many different values of $\delta V_{\max }$, the experimental data (plotted in a log-log plot) fitted well to the theoretical straight line.

To our best knowledge the power law predicted by OGY has never been tested experimentally. This may be due to the hardship involved in the required experiments; for example, quite a large number of measurements should be done, the waiting period between perturbations could be extremely long, the noise-to-signal ratio could be too large, etc. The copperphosphoric acid system, however, seems to be an ideal tool for testing the power law. The average period of oscillations varies in the order of seconds and the noise-to-signal ratio is small enough. The only drawback is the slow drift of the ever aging system. This problem was circumvented by the following "validating" procedure. In principle, we track the shift of the dynamical state in the bifurcation diagram and readjust the control parameters in such a way that the system is again placed in the desired chaotic range. To minimize the number of measurements for tracking the drift, we have chosen a reference point in the bifurcation diagram that can be easily checked upon. Our choice was the bifurcation point corresponding to the transition from period-two to period-four oscillations.

The validating procedure was applied to a given system as follows. After finding the parameter values (rotation rate and potential) giving the desired chaotic behavior, we also determined the potential value $V_{\mathrm{R}}$ corresponding to our reference point. In every $15 \mathrm{~min}$ of a typically 7-h-long series of experiments, we decreased the potential to $V_{R}$ and adjusted the rotation rate such that the system showed a transition from period-four to period-two oscillations. Then the potential was set back to its original value giving us again the desired chaotic behavior. We note that by the end of a day-long series of experiments only a total of $60 \mathrm{rpm}$ correction had to be applied to compensate for the drift of the system.

The average length of chaotic transients $\langle\tau\rangle$ for a given value of $\delta V_{\max }$ has been calculated from $\tau$ values determined for fifteen randomly selected initial conditions. The value of $\tau$ for one control session, the time elapsed between turning on and achieving control is determined as follows. We declare that control has been successful if the next-return values remain within a small region of the fixed point for at least five successive cycles. The transient time $\tau$ is then calculated as the number iterates on the map (or the number of cycles) taken between turning control on and achieving the first successful iterate. Once the unstable period-one orbit has been stabilized for five cycles, we turn control off and let the system wander chaotically for the next fifteen cycles before control is turned on again. This procedure is repeated fifteen times at a given value of $\delta V_{\max }$. Since the orbit on the uncontrolled chaotic attractor is ergodic, we may assume that the thus determined $\tau$ values correspond to chaotic transients from randomly selected initial conditions.

The average transient time $\langle\tau\rangle$ has been determined for eight different $\delta V_{\max }$ values varied between 0.13 and $0.5 \mathrm{mV}$. In Figure 9 we show the results in a $\ln -\ln$ plot. The value of exponent $\gamma=1.37 \pm 0.05$ is the slope of the line calculated by

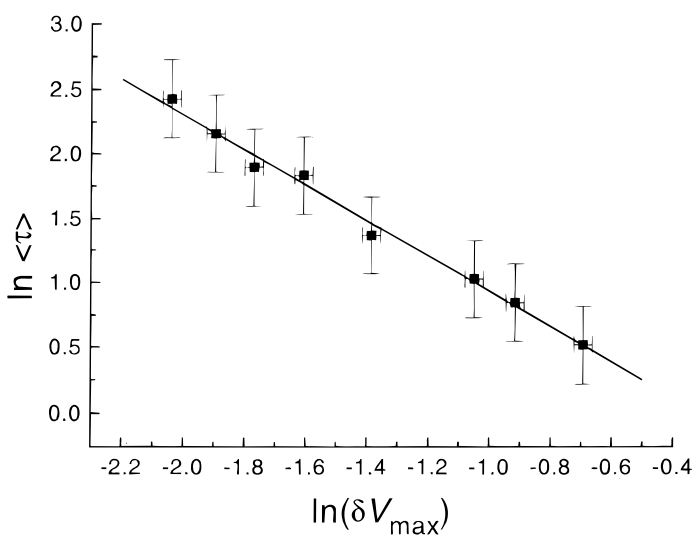

Figure 9. Average time to achieve control $\langle\tau\rangle$ as a function of maximum perturbation $\delta V_{\max }$ allowed during control. The anodic potential applied to the uncontrolled system is $509 \mathrm{mV}$ and total ohmic resistance is $203 \Omega$. The rotation rate has been varied between 1724 and $1752 \mathrm{rpm}$ as required by the so-called "validating" process described in the text.

a linear least-squares fit. Although, the error bars indicate small variations about the general power-law trend, one may say that the agreement is good. Such variations have also been found in numerical calculations ${ }^{3}$ and are, most probably, due to the fractal nature of the chaotic attractor.

Application of the SPF algorithm assumes that the system dynamics can be represented by a 1D next-return map for which $\lambda_{\mathrm{s}} \approx 0$ and thus $\gamma \approx 1$. This has been tested for the logistic map $x_{n+1}=a x_{n}\left(1-x_{n}\right)$ with $a=3.8$ by using a total of 30000 randomly selected initial points for an appropriate range of $\delta a_{\max }$ values. The calculated data fitted well to a straight line of slope $\gamma=1.09 \pm 0.04$. The fact that the experimentally determined $\gamma$ value is considerably larger than one is indicative of the fractal dimension of the map, which also follows from the fractal nature of the chaotic attractor (correlation dimension is 2.25). However, our experiments prove that the fractal character of the next-return map does not preclude the successful application of the control algorithm to the copper-phosphoric acid system.

\section{Conclusion}

In conclusion, we have shown first numerically and then experimentally that a simple proportional feedback algorithm can be successfully applied to control the chaotic current oscillations during electrodissolution of a rotating disk copper electrode into phosphoric acid electrolyte. We have stabilized the unstable period-one and period-two orbits embedded in the chaotic attractor by applying small perturbations to the anodic potential as the control parameter. We have done extensive experiments to test for a power-law formula relating the average chaotic transient time to the size of maximum perturbation allowed during control. To our knowledge this has been the first experimental verification of the predicted power law.

Applicability of different control algorithms to chaotic chemical systems has not yet been fully tested. The copperphosphoric acid system appears to be an ideal playground for the following reasons: (i) the reproducibility of the experiments is good, (ii) the level of internal noise is low, (iii) the frequency of chaotic oscillations is high, (iv) the dynamical behavior can be monitored by simple current measurement, while the control parameter (potential) is easily attainable, (v) the system shows a variety of nonlinear phenomena such as simple oscillations, period-doubling cascades, mixed-mode transitions, and chaos, and (vi) the experiments can be directly compared to the results of numerical modeling based on a simple three-variable model. 
The only drawback of the system is its drift toward equilibrium, which, however, can be dramatically slowed by applying low temperature. In addition, we have shown that a validating procedure can be effectively applied to compensate for the unavoidable shift. We plan to investigate the application of different control algorithms to the present system. Experiments to achieve control of higher periodic orbits or mixed-mode transitions and to stabilize unstable fixed points using derivative control strategy ${ }^{21}$ are also underway.

Acknowledgment. This work has been supported by the following Hungarian Research Grants: OTKA 17784, MKM 236, and AKP 96-145/20. We thank Marc T. M. Koper and John L. Hudson for helpful discussions.

\section{References and Notes}

(1) Hudson, J. L.; Tsotsis, T. T. Chem. Eng. Sci. 1994, 49, 1493.

(2) Koper, M. T. M. Adv. Chem. Phys. 1996, 92, 161.

(3) Ott, E.; Grebogi, C.; Yorke, J. A. Phys. Rev. Lett. 1990, 64, 1196.

(4) Shinbrot, T. Adv. Phys. 1995, 44, 73. 240 .
(6) Schneider, F. W.; Blittersdorf, R.; Förster, A.; Hauck, T.; Lebender, D.; Müller, J. J. Phys. Chem. 1993, 97, 12244.

(7) Lekebusch, A.; Förster, A.; Schneider, F. W. J. Phys. Chem. 1995 99, 681

(8) Parmananda, P.; Sherard, P.; Rollins, R. W.; Dewald, H. D. Phys. Rev. E 1993, 47, R3003.

(9) Parmananda, P.; Rollins, R. W.; Sherard, P.; Dewald, H. D. in Proceedings of the 2nd Conference on EXPERIMENTAL CHAOS, Ditto, W.; Pecora, L.; Vohra, S.; Shlesinger, M.; Spano, M., Eds.; Word Scientific: River Ridge, NJ, 1995; pp 304-316.

(10) Albahadily, F. N.; Schell, M. J. Chem. Phys. 1988, 88, 4312.

(11) Albahadily, F. N.; Ringland, J.; Schell, M. J. Chem. Phys. 1989, $90,813$.

(12) Schell, M.; Albahadily, F. N. J. Chem. Phys. 1989, 90, 822.

(13) Koper, M. T. M.; Gaspard, P. J. Chem. Phys. 1992, 96, 7797.

(14) Peng, B.; Petrov, V.; Showalter, K. J. Phys. Chem. 1991, 95, 4957.

(15) Petrov, V.; Peng, B.; Showalter, K. J. Chem. Phys. 1992, 96, 7506.

(16) Parmananda, P. Electrochim. Acta 1995, 41, 377.

(17) Koper, M. T. M.; Gaspard, P. J. Phys. Chem. 1991, 95, 4945.

(18) Kiss, I. Z.; Gáspár, V.; Nyikos, L. J. Phys. Chem., submitted.

(19) Vidal, R.; West, A. C. J. Electrochem. Soc. 1995, 142, 2682.

(20) Letellier, C.; Le Sceller, L.; Dutertre, P.; Gouesbet, G.; Fei, Z.; Hudson, J. L. J. Phys. Chem. 1995, 99, 7016.

(21) Parmananda, P.; Eiswirth, M. J. Phys. Chem. 1996, 100, 16568. 